\title{
The association between cognition and dual-tasking among older adults: the effect of motor function type and cognition task difficulty
}

This article was published in the following Dove Medical Press journal:

Clinical Interventions in Aging

\author{
Hossein Ehsani ${ }^{1,2}$ \\ Martha Jane Mohler ${ }^{1-3}$ \\ Kathy O'Connor ${ }^{4}$ \\ Edward Zamrini ${ }^{4,5}$ \\ Coco Tirambulo ${ }^{2}$ \\ Nima Toosizadeh ${ }^{1-3}$ \\ 'Department of Biomedical \\ Engineering, University of Arizona, \\ Tucson, AZ, USA; ${ }^{2}$ Arizona Center \\ on Aging, Department of Medicine, \\ University of Arizona, Tucson, AZ, \\ USA; ${ }^{3}$ Division of Geriatrics, General \\ Internal Medicine and Palliative \\ Medicine, Department of Medicine, \\ University of Arizona, Tucson, AZ, \\ USA; ${ }^{4}$ Neurology Department, Banner \\ Sun Health Research Institute, Sun \\ City, AZ, USA; ${ }^{5}$ Department of \\ Neurology, University of Utah, Salt \\ Lake City, UT, USA
}

Correspondence: Hossein Ehsani University of Arizona, Bioscience Research Building, I60I E Helen St., Tucson, AZ 857I9, USA

Tel +I 5206268780

Fax + I 52062658 II

Email hehsani@aging.arizona.edu
Background: Dual-task actions challenge cognitive processing. The usefulness of objective methods based on dual-task actions to identify the cognitive status of older adults has been previously demonstrated. However, the properties of select motor and cognitive tasks are still debatable. We investigated the effect of cognitive task difficulty and motor task type (walking versus an upper-extremity function [UEF]) in identifying cognitive impairment in older adults. Methods: Older adults ( $\geq 65$ years) were recruited, and cognitive ability was measured using the Montreal Cognitive Assessment (MoCA). Participants performed repetitive elbow flexion under three conditions: 1) at maximum pace alone (Single-task); and 2) while counting backward by ones (Dual-task 1); and 3) threes (Dual-task 2). Similar single- and dual-task gait were performed at normal speed. Three-dimensional kinematics were measured for both motor functions using wearable sensors.

Results: One-hundred older adults participated in this study. Based on MoCA score $<20$, $21(21 \%)$ of the participants were considered cognitively impaired (mean age $=86 \pm 10$ and $85 \pm 5$ for cognitively impaired and intact participants, respectively). Within ANOVA models adjusted with demographic information, UEF dual-task parameters, including speed and range-of-motion variability were significantly higher by $52 \%$ on average, among cognitively impaired participant $(p<0.01)$. Logistic models with these UEF parameters plus age predicted cognitive status with sensitivity, specificity, and area under curve (AUC) of $71 \%, 81 \%$ and 0.77 for Dual-task 1 . The corresponding values for UEF Dual-task 2 were $91 \%, 73 \%$ and 0.81 , respectively. ANOVA results were non-significant for gait parameters within both dual-task conditions $(p>0.26)$.

Conclusion: This study demonstrated that counting backward by threes within a UEF dual-task experiment was a pertinent and challenging enough task to detect cognitive impairment in older adults. Additionally, UEF was superior to gait as the motor task component of the dual-task. The UEF dual-task could be applied as a quick memory screen in a clinical setting.

Keywords: wearable motion sensor, gait, upper-extremity function, biomechanics, MCI, Alzheimer's disease

\section{Introduction}

The need to coordinate concurrent tasks presents itself constantly in our daily life. Simultaneous performance of motor and cognitive tasks (dual-tasking) places demands on attentional resources and reflects an increasing cognitive challenge for older adults and those with cognitive impairment. Performing dual-task actions may be accompanied with a decline in the motor task performance when compared to single task performance. ${ }^{1}$ As this decline becomes more evident with increasing age, and with cognitive decline, ${ }^{1-3}$ researchers have tried to find meaningful associations between 
the state of cognition in older adults and their dual-task performance. $^{4-7}$ For instance, worsened gait performances (slower walking speed and higher gait variability) were found during dual tasking in patients with dementia. ${ }^{4,5,7,8}$ Nonetheless, the properties of select motor and cognitive tasks, and their impact on dual-task outcomes remain debatable. ${ }^{9-14}$

Previous studies have reported the potential of gaitbased dual-task assessments to reveal cognitive impairment in elderly. ${ }^{4,5,7,8}$ However, many older adults have mobility impairments and high risk of falling. ${ }^{15,16}$ In addition, there is a lack of space and time in busy clinical settings, which can affect the execution of gait testing. To resolve these issues, we previously proposed a dual-task function, based exclusively on an upper-extremity motion. ${ }^{6,17,18}$ In this method, participants performed elbow flexion/extension as quickly as possible for 20 seconds. The data obtained from this method, which is called upper-extremity function (UEF) dual-task, was employed to develop a logistic regression model to identify cognition state of participants. ${ }^{6}$ This model showed high sensitivity and specificity when compared to the Montreal Cognitive Assessment (MoCA). While gait is a daily routine activity, UEF is an uncommon motor activity and may require skill learning. Therefore, in addition to the clinical benefits of UEF over gait as the motor component of a dual-task challenge, the novelty of UEF for participants may be an additional stressor, thus more beneficial in the measurement of cognition.

Cognitive task selection is also influential in dual-task performance. ${ }^{9,13}$ The reasoning for the selection of counting numbers backward was twofold. Firstly, counting involves working memory, ${ }^{19}$ and in contrast to some other popular tasks such as naming objects/animals, is more directly related to executive function..$^{20,21}$ Secondly, since counting is a rhythmic task, it may substantially interfere with rhythmic tasks of a different frequency such as walking or repetitive elbow flexion. ${ }^{22,23}$ Previous studies have reported that in gait-based dual-task functions, the more demanding the cognitive task, the greater the decline in the motor task performance, ${ }^{13,22,24,25}$ however, the effect of cognitive task difficulty on UEF has not been previously investigated.

The purpose of the current study is to assess the impact of select motor and cognitive tasks in a dual-task challenge to test for cognitive status among older adults. To this end, we specifically examined the effect of cognitive task difficulty by comparing counting backward by ones and threes in both UEF and gait dual-tasks. Moreover, we compared the impact of motor task selection, UEF versus gait, in identifying cognition.

\section{Methods}

\section{Participants}

Community-dwelling older adults ( $\geq 65$ years) were recruited from the Banner University Medical Center in Tucson and from the Banner Sun Health Research Institute (BSHRI) in Sun City, Arizona. Participants with the following disorders were excluded: known disorders associated with severe motor deficits including stroke, diagnosed Parkinson's disease, and amputation; major mobility disorders (eg, who were unable to walk a distance of $\sim 10 \mathrm{~m}$ ); and, upper-extremity disorders (including severe bilateral shoulder or elbow rheumatoid arthritis or osteoarthritis). Assistive devices including canes and walkers were allowed, although wheelchairs were excluded. The study was approved by the University of Arizona and BSHRI Institutional Review Boards. Prior to experiments, all participants were informed about the procedure and they signed the consent form which was prepared based on the principles expressed in the Declaration of Helsinki. ${ }^{26}$ In the case of lack of clinically assessed capacity for informed consent, consent was obtained from an authorized surrogate with the assent of the participant.

\section{Clinical measurements}

Clinical measurements included: $\mathrm{MoCA}^{27}$ for cognitive impairment, the Center for Epidemiologic Studies Depression (CES-D) scale ${ }^{28}$ for depression, and the Fried index for frailty. ${ }^{29}$ As suggested by previous work, MoCA was adjusted with normative age and education level. ${ }^{30,31}$ Cognitive impairment was defined as a MoCA score less than 20 out of $30 . .^{32,33}$ Since depression is closely associated with cognitive impairment, we used the CES-D scale to control this variable. Finally, since a strong association between functional frailty and cognitive impairment was previously reported, ${ }^{34}$ we employed the Fried index to measure frailty among participants. The implemented Fried index was based on the following five criteria: unintentional weight loss, self-reported exhaustion, self-reported low physical activity, weakness (grip strength), and slow walking speed. Individuals with more than three positive criteria were considered frail, and those with one or two positive criteria were pre-frail, and those with no positive criteria were non-frail.

\section{UEF measurements}

According to our previously validated UEF method, ${ }^{6}$ wearable motion sensors (tri-axial gyroscope sensors, sample frequency $=100 \mathrm{~Hz}$, BioSensics LLC, Cambridge, MA) were employed to measure forearm and upper-arm motion, 


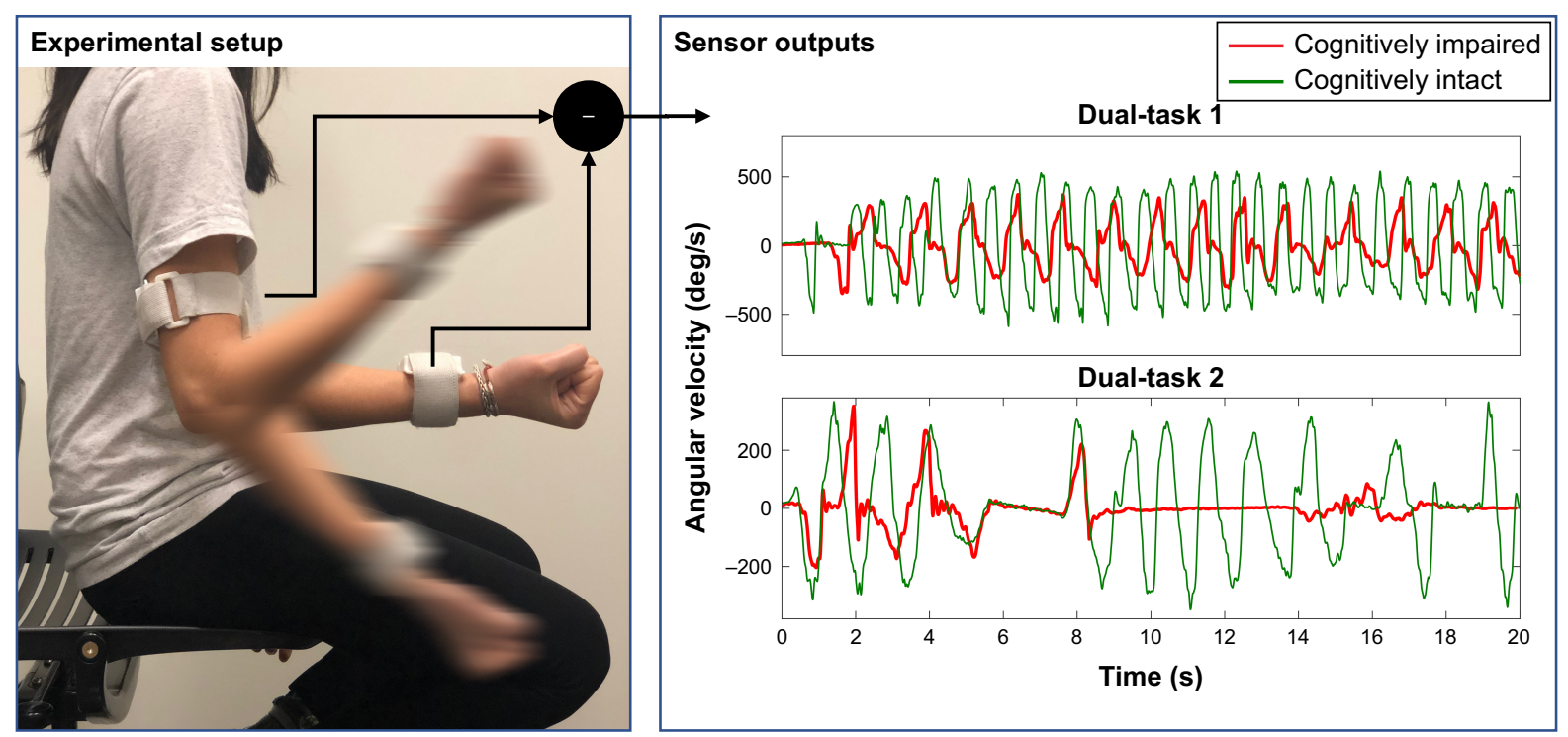

Figure I UEF experimental setup, sensor outputs and parameters: (left) Wearable motion sensors were used to capture forearm and upper-arm motion, and ultimately the elbow angular velocity during rapid elbow flexion/extension. While performing this motor task, participants counted backward by ones (Dual-task I) and threes (Dual-task 2 ) in separate experiments. (Right) Relative elbow angular velocity was obtained by subtracting sensor outputs. Results for one cognitively impaired and one cognitively intact participant during performing dual-tasks are presented.

and ultimately the elbow angular velocity (Figure 1). For the UEF test, while wearing motion sensors, each participant performed a 20 -second trial of elbow flexion in a seated position. During the test, participants repetitively and fully flexed and extended their dominant elbow as quickly as possible. The protocol was explained to participants, and they were encouraged to perform the task as fast as possible only once before each test, and no further encouragements were given to them while performing the task. Participants became familiar with the protocol by performing a short practice on their non-dominant arm prior to the actual experiment. All assessments were conducted by trained coordinators, and to assure consistency, all participants were instructed exactly the same.

Employing sensor results (Figure 1) and anthropometric information, the following UEF measures were computed: 1) speed; 2) range of motion; 3 ) power; 4) rise time; 5) speed reduction; 6) flexion number; 7) speed variability; and 8) range of motion variability (see Table 1 for definitions). Readers are referred to previous work for more details regarding UEF parameter descriptions. ${ }^{17,35}$

As shown in previous studies, the variability of a measured parameter (eg, speed) could better reflect motor performance alterations due to dual-tasking in comparison to the measured parameter itself. ${ }^{4-6}$ This variability is embodied in the coefficient of variation ( $\mathrm{CoV})$, which is defined as the ratio of standard deviation of the parameter to its mean, during the dual-task time interval. Similarly, in this study the coefficient of variation was computed for the UEF speed and range of motion parameters.

\section{Gait measurements}

An objective assessment of gait based on validated wearable sensors was employed in this study. The same sensors used to measure UEF were attached to both shins (right above the ankles) to measure three-dimensional acceleration and angular velocity of shins during gait. Participants were asked to walk at least 25 steps with their desired normal pace with which they perform everyday activities. We eliminated transient intervals of gait data (ie, initiation and termination periods), and fed the steady state part to a previously established algorithm ${ }^{36,37}$ to determine the following gait outcome measures: 1) stride velocity; 2) stride time; 3) stride length; 4) double support; 5) stride time variability; and 6) stride length variability (see Table 1 for definitions). As explained earlier for UEF, stride time and stride length variabilities were defined as $\mathrm{CoV}$ of these parameters.

\section{Dual-task protocol}

The UEF and gait tests explained in the previous sections were considered as motor task components for the dual-task experiments. Both of these single-task function tests were followed by two dual-task trials involving counting backward by either ones or threes, respectively. The motor task component of each dual-task experiment was similar to the single-task experiments; participants were asked to perform 
Table I UEF and gait parameter definition

\begin{tabular}{l|l}
\hline UEF parameters & Definition \\
\hline Speed & Mean value of elbow angular velocity range (maximum minus minimum speed) \\
\hline Range of motion & Mean value of elbow flexion range \\
\hline Power & Mean value of the product of the angular acceleration range and the range of angular velocity \\
\hline Rise time & Mean value of the required time to reach the maximum angular velocity \\
\hline Speed reduction & $\begin{array}{l}\text { Difference in angular velocity range between the last and the first } 5 \text { s of elbow flexion as a percentage of the initial } \\
\text { angular velocity range }\end{array}$ \\
\hline Flexion number & Number of flexion/extensions during $20 \mathrm{~s}$ \\
\hline Speed variability & CoV of angular velocity range during $20 \mathrm{~s}$ \\
\hline Range of motion variability & CoV of range of motion during $20 \mathrm{~s}$ \\
\hline Gait parameters & \multicolumn{2}{|l}{ Average of gait speed (horizontal distance traveled divided by duration of walking) among strides } \\
\hline Stride velocity & $\begin{array}{l}\text { Time interval starts when one foot makes contact with the ground and ends when that same foot contacts the } \\
\text { ground again }\end{array}$ \\
\hline Stride time & Distance traveled by the same limb between two successive heel contacts \\
\hline Stride length & $\begin{array}{l}\text { Duration of the initial and terminal double support when both feet are in contact with the ground as a percentage } \\
\text { of the stride time }\end{array}$ \\
\hline Double support & CoV of gait stride velocity among strides \\
\hline Stride velocity variability & CoV of gait stride time among strides \\
\hline Stride time variability & CoV of gait stride length among strides \\
\hline Stride length variability & $\begin{array}{l}\text { Note: Definitions for UEF parameters adapted from Toosizadeh et al; }{ }^{18} \text { definitions for gait parameters adapted from Najafi et al. }{ }^{37} \\
\text { Abbreviation: CoV, coefficient of variation. }\end{array}$ \\
\hline
\end{tabular}

the arm flexion/extension as quickly as possible and the gait test in the normal desired speed. Participants rested enough for about 5 minutes between the trials. To minimize falling risk among participants, they only performed normal paced walking, rather than fast walking tests. On the other hand, since UEF is an established protocol for frailty measurement, the rapid elbow flexion/extension procedure was not altered in this study. Of note, to better represent the natural environment in performing daily activities, no instruction was given to prioritize either the motor task or counting task.

Counting backward by ones is simple enough for older adults to perform, and when combined with a specific motor task (ie, UEF or gait), it has been proven effective to identify older adults with impaired cognition. ${ }^{6}$ Within the current experimental setup, participants were asked to combine counting backward by three with both UEF and walking. Of note, we previously asked healthy young participants to perform the UEF while counting backward by sevens, and found the dual-task so arduous that the UEF motion was replete with pauses; hence, counting backward by sevens was not considered here.

For all dual-task trials, we calculated the UEF and gait parameters (see above). Using the corresponding values obtained from the single-task trials, we also computed dualtask cost as the percentage change in each UEF and gait parameter between dual-task and single-task conditions. Moreover, we computed the number of correctly counted numbers by subtracting the number of mistakes in reverse counting from the total counted numbers. This parameter is an indication of the speed and accuracy of the secondary task (ie, counting backward) within the dual-task condition.

Henceforth, the combination of UEF with counting backward by ones and threes has been designated as "UEF Dual-task 1" and "UEF Dual-task 2", respectively. Similarly, we have used "Gait Dual-task 1" and "Gait Dual-task 2" for the walking task while counting backward by ones and threes.

\section{Statistical analysis}

Based on MoCA scores participants were divided into two groups of cognitively intact and cognitively impaired. Performing separate multivariable analysis of variance (ANOVA) models, we compared UEF parameters (dependent variable) among these two groups (independent variable), in both dual-task trials. As frailty and cognitive status could have a strong association, in addition to age, body mass index (BMI), and sex, frailty status of the participants was considered as a covariate in these analyses. Cohen's effect size $(d)$ was calculated in each analysis.

Using the same procedure explained previously ${ }^{18}$ within each dual-task condition a logistic regression model was 
developed to predict cognitive status via the select demographic and UEF parameters. Briefly, the following steps were taken to construct logistic models: 1) descriptive analysis of UEF parameters: outliers detection within each cognition group using box plots and histograms and testing of distribution normality using Shapiro-Wilk W test; 2) univariate analysis for UEF parameters as independent variables: UEF parameters with significant association with MoCA were selected for subsequent steps; 3) testing of collinearity between UEF parameters: using variance inflation factor (VIF) values. A VIF cutoff value larger than 10 was considered an indication of collinearity; ${ }^{38}$ and 4) stepwise parameter selection: UEF and demographic parameters were selected based on Akaike information criterion (AIC) values.

The same statistical analysis was employed for the data obtained from gait experiments. For all models, sensitivity, specificity, and area under curve (AUC) were estimated using receiver operator characteristic (ROC) curves for identifying cognitively impaired participants. The summary of the results is presented as mean (standard deviation - Std. Dev.). All analyses were done using JMP (Version 11; SAS Institute Inc., Cary, NC, USA), and statistical significance was concluded when $p<0.05$.

\section{Results \\ Participants}

A total of 100 older adults participated in this study. Based on MoCA; 21 (21\%) participants were diagnosed with cognitive impairment with a mean age and BMI of 86 (10) years and
$26.32(4.57) \mathrm{kg} / \mathrm{m}^{2}$, respectively; corresponding values were 85 (5) years and $26.01(4.16) \mathrm{kg} / \mathrm{m}^{2}$ for the cognitively intact group. Neither age nor the depression score was associated with the cognitive status of the participants $(p>0.13)$. However, frailty was significantly associated with the cognition status ( $p=0.03)$. Further, none of the recruited participants had difficulty understanding questions and counting numbers in English, as all of them were English speakers. All sociodemographic data and clinical information are reported in Table 2. Although all participants managed to perform both UEF dual-task trials, 8 ( 6 cognitively impaired) of them could not finish gait dual-tasks successfully, due to deconditioning or instability. These participants were excluded in the ANOVA models corresponding to gait.

\section{ANOVA results}

As shown in Table 3, when adjusted for age, BMI, sex, and frailty status, none of the UEF parameters were significantly different between the groups for the single task function $(p>0.12)$. Within both UEF dual-task functions, speed variability and range of motion variability were significantly associated with the cognitive status of the participants ( $p<0.01$, Figure 2); among dual-task conditions on average, the maximum effect sizes were also observed within these two parameters $(d \geq 0.36$ for speed variability and $d \geq 0.34$ for range of motion variability). All other UEF parameters, except for speed reduction and power ( $p \geq 0.06)$, were significantly different between the groups within both dual-task functions (Table 3). Within both UEF dual-tasks, the cost of all parameters, except the range of motion variability

Table 2 Sociodemographic and clinical measures

\begin{tabular}{|c|c|c|c|c|c|c|}
\hline & Male & Female & Total & $\begin{array}{l}\text { Cognitively intact } \\
(\mathrm{MoCA} \geq 20)\end{array}$ & $\begin{array}{l}\text { Cognitively impaired } \\
(\text { MoCA }<20)\end{array}$ & $p$-value ${ }^{\dagger}$ \\
\hline Number (\% of the group) & $39(39)$ & $61(6 \mathrm{I})$ & 100 & $79(79)$ & $2 I(2 I)$ & $0.26^{t \dagger}$ \\
\hline Age, year (Std. Dev.) & $84.31(5.93)$ & $86.03(6.24)$ & $85.36(6.15)$ & $85.07(4.84)$ & $86.43(9.72)$ & 0.37 \\
\hline Stature, cm (Std. Dev.) & $173.93(8.61)$ & $160.32(7.16)$ & $165.62(10.20)$ & $166.17(10.40)$ & $163.58(9.37)$ & 0.31 \\
\hline Body mass, kg (Std. Dev.) & $78.14(14.67)$ & $67.73(12.90)$ & $71.79(14.48)$ & 72.09 (14.49) & $70.66(14.73)$ & 0.70 \\
\hline BMI, kg/m² (Std. Dev.) & $25.79(4.24)$ & $26.26(4.25)$ & $26.07(4.23)$ & $26.01(4.16)$ & $26.32(4.57)$ & 0.76 \\
\hline MoCA score, 0-30 (Std. Dev.) & $23.72(3.22)$ & $23.85(3.6 \mathrm{I})$ & $23.8(3.45)$ & $25.23(2.25)$ & I8.43 (0.87) & $<0.000 I^{*}$ \\
\hline CES-D score, 0-30 (Std. Dev.) & $3.22(4.01)$ & $3.38(3.65)$ & $3.32(3.77)$ & $3.16(3.77)$ & $4.69(3.65)$ & 0.13 \\
\hline \multicolumn{6}{|l|}{ Frailtyttt } & $0.03 *$ \\
\hline Non-frail, n (\% of group) & $12(31)$ & $7(11)$ & $19(19)$ & $18(23)$ & I (4) & - \\
\hline Pre-frail, n (\% of group) & $16(4 I)$ & $44(72)$ & $60(60)$ & $50(63)$ & $10(48)$ & - \\
\hline Frail, n (\% of group) & II (28) & $10(17)$ & $2 I(2 I)$ & II (14) & $10(48)$ & - \\
\hline
\end{tabular}

Notes: ${ }^{\dagger} p$-values are reported for differences between cognitively intact and cognitively impaired groups. ${ }^{\dagger} \mathrm{H}$ Sex was considered as the independent variable. ${ }^{\mathrm{t} t \mathrm{t} F r a i l t y}$ was considered as an ordinal variable. *Significant difference.

Abbreviations: BMI, body mass index; MoCA, Montreal Cognitive Assessment; CES-D, Center for epidemiologic studies depression. 
Table 3 Mean (standard deviation) of UEF parameters, by cognition status

\begin{tabular}{|c|c|c|c|c|}
\hline Single task & $\begin{array}{l}\text { Cognitively intact } \\
(M o C A \geq 20)\end{array}$ & $\begin{array}{l}\text { Cognitively impaired } \\
(\mathrm{MoCA}<20)\end{array}$ & $p$-value ${ }^{\dagger}$ & Effect size \\
\hline Speed (deg/s) & $827.34(289.05)$ & $620.87(216.28)$ & 0.12 & 0.31 \\
\hline Power $\left(\mathrm{deg}^{2} / \mathrm{s}^{3} \times 10^{7}\right)$ & $123.85(111.11)$ & $69.53(55.87)$ & 0.42 & 0.22 \\
\hline Range of motion (deg) & $98.17(27.02)$ & $83.91(25.90)$ & 0.13 & 0.22 \\
\hline Rise time $(s)$ & $0.55(2.54)$ & $0.34(0.09)$ & 0.64 & 0.04 \\
\hline Speed reduction (\%) & $1.53(12.95)$ & $3.74(11.57)$ & 0.40 & 0.07 \\
\hline Speed variability (\%) & $11.12(6.34)$ & $12.03(4.8 I)$ & 0.95 & 0.06 \\
\hline Range of motion variability (\%) & $11.55(8.12)$ & $12.73(6.29)$ & 0.98 & 0.06 \\
\hline Flexion number $(\mathrm{n})$ & $23.09(8.86)$ & $17.43(5.50)$ & 0.16 & 0.28 \\
\hline \multicolumn{5}{|l|}{ Dual-task I } \\
\hline Speed (deg/s) & $762.23(250.5)$ & $549.90(202.07)$ & $0.03^{*}$ & 0.36 \\
\hline Power $\left(\mathrm{deg}^{2} / \mathrm{s}^{3} \times 10^{7}\right)$ & $99.50(90.25)$ & $51.22(41.45)$ & 0.18 & 0.24 \\
\hline Range of motion (deg) & $99.06(25.85)$ & $76.28(28.82)$ & $0.003 *$ & 0.35 \\
\hline Rise time (s) & $0.29(0.10)$ & $0.38(0.17)$ & $0.05^{*}$ & 0.31 \\
\hline Speed reduction (\%) & $5.47(8.57)$ & $6.54(8.36)$ & 0.06 & 0.05 \\
\hline Speed variability (\%) & $11.05(3.33)$ & $14.93(5.62)$ & $<0.00 I^{*}$ & 0.4 \\
\hline Range of motion variability (\%) & $9.99(5.03)$ & $15.28(9.68)$ & $<0.01 *$ & 0.34 \\
\hline Flexion number $(\mathrm{n})$ & $20.73(6.38)$ & $16.57(5.92)$ & 0.24 & 0.27 \\
\hline \multicolumn{5}{|l|}{ Dual-task 2} \\
\hline Speed (deg/s) & $646.79(241.15)$ & $485.87(186.43)$ & $0.05^{*}$ & 0.28 \\
\hline Power $\left(\mathrm{deg}^{2} / \mathrm{s}^{3} \times 10^{7}\right)$ & 71.59 (70.67) & $42.61(44.17)$ & 0.32 & 0.18 \\
\hline Range of motion (deg) & $96.02(30.26)$ & $70.67(24.40)$ & $0.006 *$ & 0.35 \\
\hline Rise time (s) & $0.38(0.15)$ & $0.52(0.30)$ & $0.04 *$ & 0.30 \\
\hline Speed reduction (\%) & $7.63(10.27)$ & $10.46(20.2)$ & 0.26 & 0.09 \\
\hline Speed variability (\%) & $|3.5|(4.90)$ & $19.62(12.04)$ & $<0.00 I^{*}$ & 0.36 \\
\hline Range of motion variability (\%) & $10.13(7.25)$ & $17.64(8.21)$ & $<0.01^{*}$ & 0.41 \\
\hline Flexion number $(n)$ & $16.24(6.41)$ & $13.10(6.10)$ & 0.44 & 0.20 \\
\hline
\end{tabular}

Notes: ${ }^{\dagger}$ Models were adjusted with age, BMI, sex, and frailty status. *Significant difference.

Abbreviation: MoCA, Montreal Cognitive Assessment.

$(p<0.04)$, were non-significant $(p>0.05)$ between the groups. On the contrary to UEF, as shown in Table 4, gait parameters were not significantly different in Dual-task 1 $(p>0.41)$ and Dual-task $2(p>0.26)$ trials. Also, none of the cost variables corresponding to the gait parameters were significantly different between the groups.

Importantly, differences in UEF parameters in both UEF dual-tasks among cognitively intact and cognitively impaired

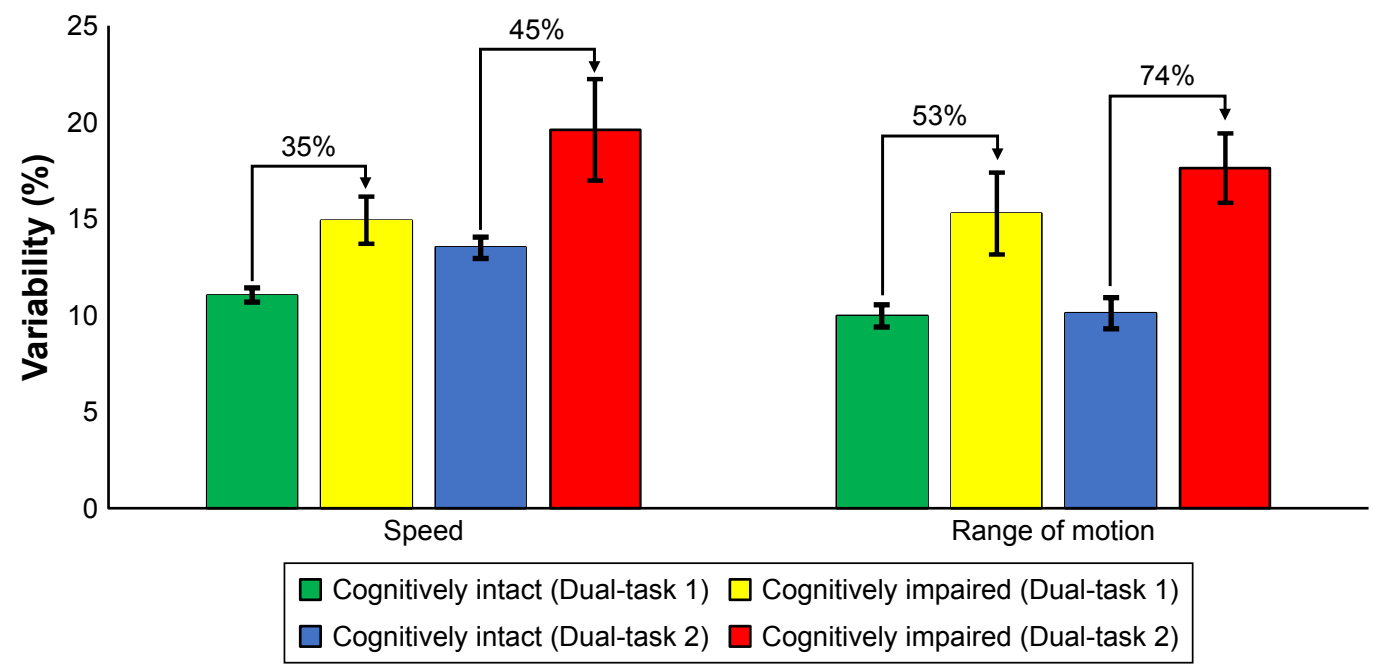

Figure 2 Comparison of speed variability and range of motion variability among cognitively intact and cognitively impaired participants, within UEF Dual-task I and Dual-task 2. 
Table 4 Mean (standard deviation) of gait parameters, by cognition status

\begin{tabular}{|c|c|c|c|c|}
\hline Single task & $\begin{array}{l}\text { Cognitively intact } \\
(M o C A \geq 20)\end{array}$ & $\begin{array}{l}\text { Cognitively impaired } \\
(\text { MoCA }<20)\end{array}$ & $p$-value ${ }^{\dagger}$ & Effect size \\
\hline Stride velocity $(\mathrm{m} / \mathrm{s})$ & $0.91(0.33)$ & $0.94(0.3 \mathrm{I})$ & 0.31 & 0.03 \\
\hline Stride time (s) & $1.17(0.21)$ & $1.26(0.38)$ & 0.24 & 0.14 \\
\hline Stride length $(m)$ & $1.02(0.31)$ & $1.02(0.24)$ & 0.38 & 0.01 \\
\hline Double support (\%) & $23.28(7.73)$ & $25.21(7.77)$ & 0.94 & 0.10 \\
\hline Stride velocity variability (\%) & $|2.5|(8.4 \mid)$ & I 7.07 (I4.49) & 0.14 & 0.19 \\
\hline Stride time variability (\%) & $0.072(0.079)$ & $0.20(0.32)$ & 0.06 & 0.32 \\
\hline Stride length variability (\%) & $0.10(0.083)$ & $0.13(0.13)$ & 0.35 & 0.13 \\
\hline \multicolumn{5}{|l|}{ Dual-task I } \\
\hline Stride velocity $(\mathrm{m} / \mathrm{s})$ & $0.82(0.36)$ & $0.83(0.34)$ & 0.86 & 0.01 \\
\hline Stride time (s) & $1.28(0.30)$ & $\mathrm{I} .44(0.59)$ & 0.41 & 0.17 \\
\hline Stride length $(m)$ & $0.97(0.32)$ & $0.98(0.29)$ & 0.41 & 0.01 \\
\hline Double support (\%) & $25.96(9.26)$ & $28.27(9.95)$ & 0.82 & 0.09 \\
\hline Stride velocity variability (\%) & $14.37(9.40)$ & $17.14(12.41)$ & 0.82 & 0.10 \\
\hline Stride time variability (\%) & $0.09(0.13)$ & $0.19(0.33)$ & 0.48 & 0.21 \\
\hline Stride length variability (\%) & $0.10(0.081)$ & $0.12(0.10)$ & 0.70 & 0.09 \\
\hline \multicolumn{5}{|l|}{ Dual-task 2} \\
\hline Stride velocity $(\mathrm{m} / \mathrm{s})$ & $0.77(0.38)$ & $0.76(0.34)$ & 0.64 & 0.01 \\
\hline Stride time (s) & $1.44(0.54)$ & $1.39(0.30)$ & 0.39 & 0.04 \\
\hline Stride length $(m)$ & $0.95(0.34)$ & $0.94(0.32)$ & 0.54 & 0.01 \\
\hline Double support (\%) & $28.10(11.07)$ & $29.03(8.85)$ & 0.47 & 0.03 \\
\hline Stride velocity variability (\%) & $14.93(10.42)$ & $16.13(9.79)$ & 0.65 & 0.04 \\
\hline Stride time variability (\%) & $0.12(0.2 \mathrm{I})$ & $0.11(0.17)$ & 0.26 & 0.02 \\
\hline Stride length variability (\%) & $0.10(0.085)$ & $0.12(0.075)$ & 0.82 & 0.09 \\
\hline
\end{tabular}

Notes: ${ }^{\top}$ Models were adjusted with age, BMI, sex and frailty status.

Abbreviation: MoCA, Montreal Cognitive Assessment.

groups based on MoCA. Mean (Std. Dev.) values are presented in Table 3. The effect size is presented for each variable.

Differences in gait parameters in both dual-tasks among cognitively intact and cognitively impaired groups based on MoCA. Mean (Std. Dev.) values are presented in Table 4. The effect size is presented for each variable.

\section{Logistic models}

For the logistic models corresponding to the UEF dual-tasks, speed variability and range of motion variability, in addition to age, were selected as independent variables. Results from UEF Dual-task 1 logistic regression model identified cognitive impairment (based on MoCA) with a sensitivity and specificity of $71 \%$ and $81 \%$ (AUC $=0.77$ ). Similarly, the logistic model based on the UEF Dual-task 2 demonstrated $91 \%$ and $73 \%$ sensitivity and specificity, respectively (AUC $=0.81$, Table 5). The corresponding ROC curves are depicted in Figure 3.

To compare gait and UEF, we only considered those participants who could perform both motor functions and

Table 5 Results of the multivariable logistic regression models for the UEF dual-task functions

\begin{tabular}{l|l|l|l|l|l|l}
\hline UEF Dual-task I & $\begin{array}{l}\text { Parameter } \\
\text { estimates }\end{array}$ & $\begin{array}{l}\text { Standard } \\
\text { errors }\end{array}$ & $\chi^{2}$ & $\boldsymbol{p}$-value & $\mathbf{9 5 \%} \mathbf{C I}$ lower & $\mathbf{9 5 \%} \mathbf{C l}$ upper \\
\hline Intercept & 10.04 & 4.18 & 5.76 & $0.02 *$ & 2.21 & 18.8 \\
Age & -0.068 & 0.047 & 2.07 & 0.15 & -0.16 & 0.022 \\
Speed variability & -0.15 & 0.077 & 3.93 & $0.04^{*}$ & -0.31 & -0.0079 \\
Range of motion variability & -0.085 & 0.0487 & 3.02 & 0.08 & -0.18 & 0.0089 \\
\hline UEF Dual-task 2 & 6.17 & 3.74 & 2.72 & 0.10 & -0.95 & 13.90 \\
\hline Intercept & -0.031 & 0.042 & 0.54 & 0.46 & -0.12 & 0.051 \\
Age & -0.070 & 0.046 & 2.32 & 0.12 & -0.17 & 0.013 \\
Speed variability & -0.081 & 0.035 & 5.28 & $0.02 *$ & -0.16 & -0.014 \\
Range of motion variability
\end{tabular}

Note: *Significant difference. 


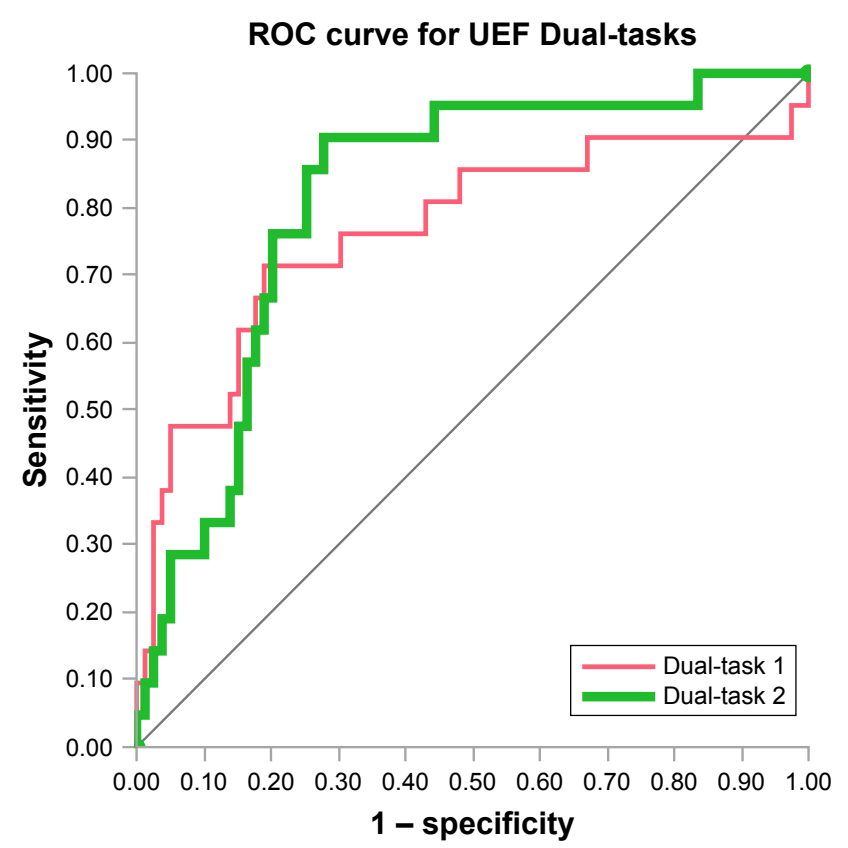

Figure 3 The ROC curves of the UEF logistic models predicting cognitive status. Models were developed from all participants while counting backward by ones (ie, Dual-task I) and threes (ie, Dual-task 2).

built the corresponding logistic models for Dual-task 1 and Dual-task 2. The gait models were constructed using stride time variability, stride length variability, and age as the independent variables. For gait models, AUC values of 0.73 for Dual-task 1, and 0.66 for Dual-task 2 were obtained.
The AUC for the corresponding models for UEF were 0.81 and 0.79 for Dual-task 1 and Dual-task 2, respectively. The corresponding ROC curves are depicted in Figure 4.

\section{Discussion}

The usefulness of objective dual-task methods to identify the cognitive status of older adults has been previously demonstrated. However, the properties of select motor and cognitive tasks, and their impact on predicting cognitive impairment were unclear. In this study, we investigated two motor tasks including normal walking speed and a novel UEF task, and two cognitive task difficulties using counting backward by ones and threes.

Focusing on UEF, results of this study showed that counting backward by threes as the cognitive component of the dual-task experiment is a sufficiently challenging task to reveal the cognitive status of older adults, and sensitive to those at the earlier stages of cognitive decline (Figure 3 ). Although the efficiency of counting backward by ones has also previously been demonstrated for this purpose, ${ }^{6}$ by comparing the performance indicators of the logistic models obtained from UEF dual-task actions, one can discern that the more difficult task of counting backward by threes is a more sensitive method to identify cognitively impaired older adults (91\% for UEF Dual-task 2 in comparison to $71 \%$ for UEF Dual-task 1). Despite this improvement, increasing the
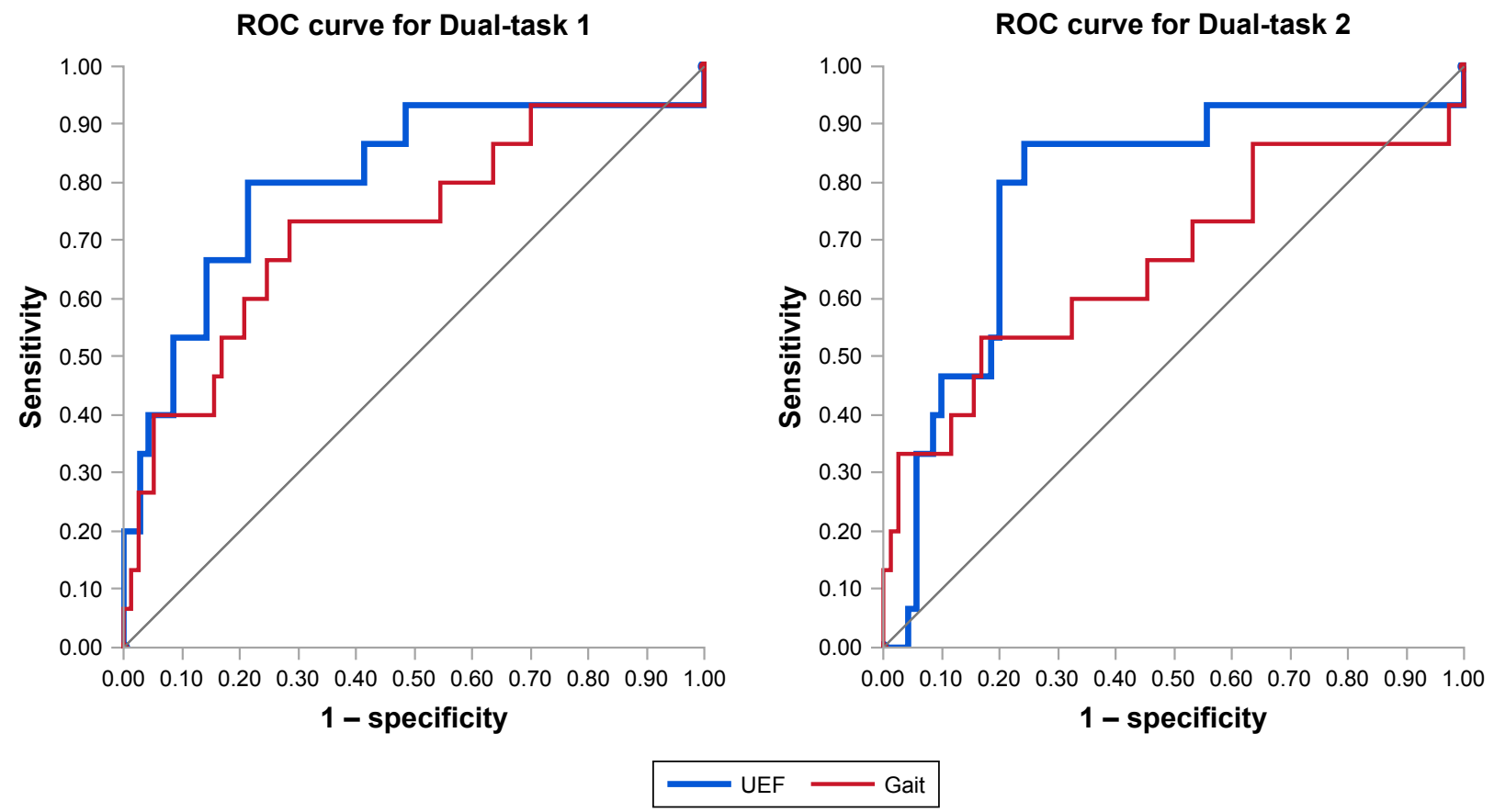

Figure 4 The ROC curves of the UEF and gait logistic models predicting cognitive status. Models were developed based on the results obtained from those participants who were able to perform both motor functions while counting backward by ones (ie, Dual-task I) and threes (ie, Dual-task 2). 
cognitive task difficulty caused a decrement in the specificity for the corresponding logistic model (81\% for UEF Dualtask 1 versus $73 \%$ for UEF Dual-task 2). For the purpose of early screening of cognitive impairment, high sensitivity is desired, and specificity (the statistical probability that an individual who is not cognitively impaired will be correctly identified as negative) is less critical.

Another important feature of a dual-task function that may help to optimize its efficacy for identifying cognitively impaired elderly is related to the motor task type. As we have previously discussed, ${ }^{6}$ using the UEF dual-task over gait dual-task is beneficial for clinical applications, especially in the case of mobility impairments and high fall risk. In this study, performing UEF was feasible among all the participants, while only $71 \%$ of cognitively impaired participants were able to finish the gait dual-task functions. In addition to this advantage, the UEF method provides a more sensitive and specific logistic model for predicting cognitive status in comparison to gait (Figure 4). Because walking is a routine daily activity, minimum skill learning is involved in its performance. Whereas the UEF involves performing a novel and unfamiliar movement, which may require more motor cortex activation. ${ }^{39}$ Of note, here the role of motor cortex in performing gait is not ignored, ${ }^{40}$ and the emphasis is on the degree in which the motor cortex is stimulated. On the other hand, working memory is primarily involved in learning a new skill, ${ }^{41}$ and counting backward relies on working memory. ${ }^{42}$ Consequently, results suggest that during UEF Dual-task 2 working memory was more challenged. Previous work has suggested working memory decline in cognitively impaired elders, ${ }^{43}$ accordingly, the hypothetical explanation for our observations here is that combination of skill-learning (UEF) and counting backward by threes would create a stress test for the brain to reveal its impairment. Although this explanation seems plausible, it requires a rigorous confirmation via brain imaging (eg, fMRI) and neuropsychiatric correlates in future studies.

In this study, gait parameters were not significantly different between the groups in neither of dual-task trials. One potential reason for such results is the small number of cognitively impaired participants (15 participants) who could perform the gait dual-task trials. Moreover, as suggested in previous works, dual-task gait trials may not detect cognitive impairments among frail older adults. ${ }^{10,12}$ Of note, in the current study, frailty (as measured based on the Fried phenotype) was significantly different between the groups. It is important to note that even by adjusting the models with the frailty status, UEF parameters were significantly different between the cognitive groups. This result further underlines the potential of UEF dual-tasks for assessing cognition among community-dwelling older adults.

In our previous study, employing the same procedure within a different sample of participants, we implemented counting backward by ones while performing the repetitive elbow flexion/extension, and constructed a multivariable regression logistic model to predict cognitive status. ${ }^{6}$ Similar to the findings within our previous study, ${ }^{6}$ here we observed that variability in the motion of elbow during flexion/ extension (ie, speed variability) was more strongly associated with the cognition status compared to other parameters, such as speed itself. Within the current sample, we also observed the speed and range of motion variability as the dominant parameters in predicting cognitive status among older adults (Figure 2). Of note, within our previous work implementing counting backward by ones, the range of motion variability was not calculated as a UEF outcome. In the current study, in which we worked with a more diverse population, speed variability and range of motion variability showed significant differences among those who were cognitively impaired versus intact participants and were used to develop logistic models. Further, the results of this study showed that under dual-task function, differential gait variability was better represented using stride time variability and stride length variability when compared to stride time and stride length itself. This observation was in agreement with previous studies in which dual-task gait variability has been observed in stride time variability instead of stride time. ${ }^{4,5}$ Considering a simple analogy, one may make connections between UEF speed variability and gait stride time variability, as well as UEF range of motion variability and stride length variability.

Moreover, in contrast to our previous model, the variable of correctly counted numbers was not included in the logistic model. The comparison between different motor tasks, ie, gait and UEF, was one of the main objectives of this study; and, as gait was measured within a specific distance and not a predefined time duration, unlike UEF we did not compute the correctly counted numbers during gait. Therefore, to preserve the uniformity in the developed logistic models, and hence in the following comparisons, the "correctly counted numbers" variables were not incorporated into the logistic models.

Some limitations within this study should be acknowledged. We used MoCA, a cognitive impairment screening tool to assess cognitive status rather than clinically confirmed and neuropsychiatric and imaging determined clinically diagnosed mild cognitive impairment or dementia. In addition, we were unable to assess the type of cognitive impairment 
(eg, amnestic [pre-Alzheimer's] versus non-amnestic). In this regard, two directions should be pursued in future studies. Firstly, the performance of UEF dual-tasks should be tested among clinically confirmed groups of cognitively impaired and demented patients (eg, patients with amnestic MCI and Alzheimer's disease); and secondly, the activity of different parts of the brain should be monitored (eg, with fMRI and/or EEG) while performing UEF dual-tasks in these participants. Further, computational models of the upper-extremity musculoskeletal system ${ }^{44-46}$ may be used to examine kinetic variables (eg, elbow flexion/extension moments ${ }^{47}$ and/or muscle forces crossing this joint ${ }^{48,49}$ ) within UEF dual-tasks.

\section{Conclusion}

Within the current study, we examined the effect of motor task selection and cognitive task difficulty in a dual-task function to identify the cognitive state of community-dwelling older adults. For the motor component, we compared a daily routine activity of walking with a self-selected speed, to an unfamiliar task of rapid elbow flexion/extension. Counting backward by ones and threes were chosen as the cognitive component of the dual-tasks. Results of this study showed that the combination of UEF with counting backward by threes was a pertinent and challenging enough dual-task choice to reveal the cognitive status of older adults, especially for those who are at earlier stages of cognitive decline. Further UEF proved more sensitive than did gait as the motor task components within the dual task challenges, and better able to be performed than was gait.

\section{Acknowledgments}

This project was supported by the Centers for Disease Control and Prevention Healthy Brain Research Network (CDCHBRN). The CDC-HBRN is a Prevention Research Centers program funded by the CDC Healthy Aging ProgramHealthy Brain Initiative. The project was also supported in part by award from the National Institute of Aging (NIA) (award number: 1 R21 AG055852-01). The findings of this manuscript are those of the authors and do not necessarily represent the official views of CDC-HBRN or NIA. Thanks to coordinators for their significant contribution in coordinating study staff, and to Banner Sun Heath Research Institute's Longevity Study participants for their generous participation.

\section{Disclosure}

The authors report no conflicts of interest in this work. Mrs Kathy O’Connor, Dr Edward Zamrini, Dr Martha Jane Mohler, and Dr Nima Toosizadeh report grants from National Institute of Aging, during the conduct of the study.

\section{References}

1. Lindenberger U, Marsiske M, Baltes PB. Memorizing while walking: increase in dual-task costs from young adulthood to old age. Psychol Aging. 2000;15(3):417.

2. Verhaeghen P, Steitz DW, Sliwinski MJ, Cerella J. Aging and dual-task performance: a meta-analysis. Psychol Aging. 2003;18(3):443.

3. Camicioli R, Howieson D, Lehman S, Kaye J. Talking while walking the effect of a dual task in aging and Alzheimer's disease. Neurology. 1997;48(4):955-958.

4. Lamoth CJ, van Deudekom FJ, van Campen JP, Appels BA, de Vries OJ, Pijnappels M. Gait stability and variability measures show effects of impaired cognition and dual tasking in frail people. J Neuroeng Rehabil. 2011;8(1):2.

5. Montero-Odasso M, Casas A, Hansen KT, et al. Quantitative gait analysis under dual-task in older people with mild cognitive impairment: a reliability study. J Neuroeng Rehabil. 2009;6(1):35.

6. Toosizadeh N, Najafi B, Reiman EM, et al. Upper-extremity dual-task function: an innovative method to assess cognitive impairment in older adults. Front Aging Neurosci. 2016;8:167.

7. IJmker T, Lamoth CJ. Gait and cognition: the relationship between gait stability and variability with executive function in persons with and without dementia. Gait Posture. 2012;35(1):126-130.

8. Doi T, Shimada H, Makizako H, et al. Cognitive function and gait speed under normal and dual-task walking among older adults with mild cognitive impairment. BMC Neurol. 2014;14(1):67.

9. Dingwell JB, Robb RT, Troy KL, Grabiner MD. Effects of an attention demanding task on dynamic stability during treadmill walking. J Neuroeng Rehabil. 2008;5(1):12.

10. Kikkert LH, Vuillerme N, van Campen JP, Appels BA, Hortobágyi T, Lamoth CJ. Gait characteristics and their discriminative power in geriatric patients with and without cognitive impairment. J Neuroeng Rehabil. 2017;14(1):84.

11. Hooghiemstra AM, Ramakers IH, Sistermans N, et al. Gait speed and grip strength reflect cognitive impairment and are modestly related to incident cognitive decline in memory clinic patients with subjective cognitive decline and mild cognitive impairment: findings from the 4C study. J Gerontol Ser A. 2017;72(6):846-854.

12. Martínez-Ramírez A, Martinikorena I, Lecumberri P, et al. Dual task gait performance in frail individuals with and without mild cognitive impairment. Dement Geriatr Cogn Disord. 2016;42(1-2):7-16.

13. Qu X. Age-related cognitive task effects on gait characteristics: do different working memory components make a difference? J Neuroeng Rehabil. 2014;11(1):149.

14. Ehsani H, Tirambulo CVG, Mills CS, Zamrini E, Mohler J, Toosizadeh N. Assessing cognition using dual-task motor function: the influence of cognitive task type. Alzheimers Dement. 2018;14(7):P1444.

15. Ehsani H, Mohler J, Marlinski V, Rashedi E, Toosizadeh N. The influence of mechanical vibration on local and central balance control. J Biomech. 2018;71:59-66.

16. Toosizadeh N, Ehsani H, Miramonte M, Mohler J. Proprioceptive impairments in high fall risk older adults: the effect of mechanical calf vibration on postural balance. Biomed Eng Online. 2018;17(1):51.

17. Toosizadeh N, Mohler J, Najafi B. Assessing upper extremity motion: an innovative method to identify frailty. $J$ Am Geriatr Soc. 2015;63(6):1181-1186.

18. Toosizadeh N, Wendel C, Hsu CH, Zamrini E, Mohler J. Frailty assessment in older adults using upper-extremity function: index development. BMC Geriatr. 2017;17.

19. Lee K-M, Kang S-Y. Arithmetic operation and working memory: differential suppression in dual tasks. Cognition. 2002;83(3): B63-B68.

20. Montero-Odasso M, Bergman H, Phillips NA, Wong CH, Sourial N, Chertkow H. Dual-tasking and gait in people with mild cognitive impairment. The effect of working memory. BMC Geriatr. 2009;9(1):41.

21. Smith EE, Geva A, Jonides J, Miller A, Reuter-Lorenz P, Koeppe RA. The neural basis of task-switching in working memory: effects of performance and aging. Proc Natl Acad Sci. 2001;98(4):2095-2100. 
22. Beauchet O, Dubost V, Aminian K, Gonthier R, Kressig RW. Dualtask-related gait changes in the elderly: does the type of cognitive task matter? J Mot Behav. 2005;37(4):259.

23. Taga G, Yamaguchi Y, Shimizu H. Self-organized control of bipedal locomotion by neural oscillators in unpredictable environment. Biol Cybern. 1991;65(3):147-159.

24. Li C, Verghese J, Holtzer R. A comparison of two walking while talking paradigms in aging. Gait Posture. 2014;40(3):415-419.

25. Bock O. Dual-task costs while walking increase in old age for some, but not for other tasks: an experimental study of healthy young and elderly persons. J Neuroeng Rehabil. 2008;5(1):27.

26. Assoc WM. World medical association declaration of helsinki ethical principles for medical research involving human subjects. J Am Med Assoc. 2013;310(20):2191-2194.

27. Nasreddine ZS, Phillips NA, Bédirian V, et al. The montreal cognitive assessment, MoCA: a brief screening tool for mild cognitive impairment. J Am Geriatr Soc. 2005;53(4):695-699.

28. Orme JG, Reis J, Herz EJ. Factorial and discriminant validity of the center for epidemiological studies depression (CES-D) scale. J Clin Psychol. 1986;42(1):28-33.

29. Fried LP, Tangen CM, Walston J, et al. Frailty in older adults: evidence for a phenotype. J Gerontol A Biol Sci Med Sci. 2001;56(3): M146-M157.

30. Malek-Ahmadi M, Powell JJ, Belden CM, et al. Age-and educationadjusted normative data for the Montreal cognitive assessment (MoCA) in older adults age 70-99. Aging Neuropsychol Cogn. 2015;22(6): 755-761.

31. Measso G, Cavarzeran F, Zappala G, et al. The mini-mental state examination: normative study of an Italian random sample. Dev Neuropsychol. 1993;9(2):77-85.

32. Hancock P, Larner A. Test your memory test: diagnostic utility in a memory clinic population. Int J Geriatr Psychiatry. 2011;26(9): 976-980.

33. Larner A. Screening utility of the Montreal cognitive assessment (MoCA): in place of-or as well as-the MMSE? Int Psychogeriatr. 2012; 24(3):391-396.

34. Boyle PA, Buchman AS, Wilson RS, Leurgans SE, Bennett DA. Physical frailty is associated with incident mild cognitive impairment in community-based older persons. J Am Geriatr Soc. 2010;58(2): 248-255.

35. Ehsani H, Mohler MJ, Golden T, Toosizadeh N. Upper-extremity function prospectively predicts adverse discharge and all-cause COPD readmissions: a pilot study. Int J Chron Obstruct Pulmon Dis. 2019;14:39.

36. Aminian K, Najafi B, Büla C, Leyvraz PF, Robert P. Spatio-temporal parameters of gait measured by an ambulatory system using miniature gyroscopes. J Biomech. 2002;35(5):689-699.

37. Najafi B, Helbostad JL, Moe-Nilssen R, Zijlstra W, Aminian K. Does walking strategy in older people change as a function of walking distance? Gait Posture. 2009;29(2):261-266.
38. O'Brien RM. A caution regarding rules of thumb for variance inflation factors. Qual Quant. 2007;41(5):673-690.

39. Kawai R, Markman T, Poddar R, et al. Motor cortex is required for learning but not for executing a motor skill. Neuron. 2015;86(3):800-812.

40. Annweiler C, Beauchet O, Bartha R, et al. Motor cortex and gait in mild cognitive impairment: a magnetic resonance spectroscopy and volumetric imaging study. Brain. 2013;136:859-871.

41. Hikosaka O, Nakamura K, Sakai K, Nakahara H. Central mechanisms of motor skill learning. Curr Opin Neurobiol. 2002;12(2):217-222.

42. McLean JF, Hitch GJ. Working memory impairments in children with specific arithmetic learning difficulties. J Exp Child Psychol. 1999;74(3):240-260.

43. Gagnon LG, Belleville S. Working memory in mild cognitive impairment and Alzheimer's disease: contribution of forgetting and predictive value of complex span tasks. Neuropsychology. 2011;25(2):226-236.

44. Ehsani H, Poursina M, Rostami M, Mousavi A, Parnianpour M, Khalaf K. Efficient embedding of empirically-derived constraints in the ODE formulation of multibody systems: application to the human body musculoskeletal system. Mech Mach Theory. 2019;133:673-690.

45. Ehsani H, Rostami M, Parnianpour M. A closed-form formula for the moment arm matrix of a general musculoskeletal model with considering joint constraint and motion rhythm. Multibody Syst Dyn. 2016;36(4):377-403.

46. Ehsani H, Rostami M, Gudarzi M. A general-purpose framework to simulate musculoskeletal system of human body: using a motion tracking approach. Comput Methods Biomech Biomed Engin. 2016;19(3):306-319.

47. Rafiee S, Ehsani H, Rostami M. A PCA-assisted EMG-driven model to predict upper extremities' joint torque in dynamic movements. Paper presented at 2013 20th Iranian Conference on Biomedical Engineering (ICBME); 18-20 December, 2013; Tehran, Iran. Available from: https:// ieeexplore.ieee.org/abstract/document/6782184. Accessed March 20, 2019.

48. Moosavi F, Pasdar A, Ehsani H, Rostami M. An emg-driven musculoskeletal model to predict muscle forces during performing a weight training exercise with a dumbbell. Paper presented at 2012 19th Iranian Conference of Biomedical Engineering (ICBME); 20-21 December, 2012; Tehran, Iran. Available from: https://ieeexplore.ieee.org/abstract/ document/6519662. Accessed March 20, 2019.

49. Mousavi A, Ehsani H, Rostami M. Compliant Vs. rigid tendon models: a simulation study on precision, computational efficiency and numerical stability. Paper presented at 201421 th Iranian Conference on Biomedical Engineering (ICBME); 26-28 November, 2014. Available from: https://ieeexplore.ieee.org/abstract/document/7043900. Accessed March 20, 2019.
Clinical Interventions in Aging

\section{Publish your work in this journal}

Clinical Interventions in Aging is an international, peer-reviewed journal focusing on evidence-based reports on the value or lack thereof of treatments intended to prevent or delay the onset of maladaptive correlates of aging in human beings. This journal is indexed on PubMed Central, MedLine,

\section{Dovepress}

CAS, Scopus and the Elsevier Bibliographic databases. The manuscript management system is completely online and includes a very quick and fair peer-review system, which is all easy to use. Visit http://www.dovepress. com/testimonials.php to read real quotes from published authors. 